\title{
The Outcome of 40 Patients Becoming Pregnancy after Conservative Treatment of Cesarean Scar Pregnancy
}

\author{
Nguyen Hong Hoa ${ }^{1}$, Nguyen Thi Thanh Thao', Hoang Thi Diem Tuyet ${ }^{2}$, Van Phung Thong ${ }^{3}$ \\ ${ }^{1}$ Department of Obstetrics \& Gynecology, Faculty of Medicine, University of Medicine and Pharmacy at Ho Chi Minh City, Ho \\ Chi Minh City, Vietnam \\ ${ }^{2}$ Department of Obstetrics, Hung Vuong Hospital, Ho Chi Minh City, Vietnam \\ ${ }^{3}$ Department of Obstetrics, Tu Du Hospital, Ho Chi Minh City, Vietnam \\ Email: drhonghoa@ump.edu.vn
}

How to cite this paper: Hoa, N.H., Thao, N.T.T., Tuyet, H.T.D. and Thong, V.P. (2020) The Outcome of 40 Patients Becoming Pregnancy after Conservative Treatment of Cesarean Scar Pregnancy. Case Reports in Clinical Medicine, 9, 376-384.

https://doi.org/10.4236/crcm.2020.912052

Received: November 10, 2020

Accepted: December 15, 2020

Published: December 18, 2020

Copyright (c) 2020 by author(s) and Scientific Research Publishing Inc. This work is licensed under the Creative Commons Attribution International License (CC BY 4.0).

http://creativecommons.org/licenses/by/4.0/

\section{Open Access}

\begin{abstract}
Background: The rate of uterus is successfully conserved following the treatment of scar pregnancy which is high so pregnancy outcome following caesarean scar ectopics is getting more and more attention. Objectives: To assess pregnancy course and outcome after conservative treatment of cesarean scar pregnancy. Methods: A retrospective case series of 40 patients become pregnancy after conservative treatment of cesarean scar pregnancy by Foley or Methotrexate and aspiration. Patients in present study were treated at Hung Vuong and Tu Du Hospital between 2015 and 2017. A telephone follow-up was conducted after cesarean scar pregnancy (CSP) treatment. The outcomes of these subsequent pregnancies and mode of delivery were all recorded. Results: In 40 pregnancies, there are 22 cases of intrauterine pregnancy with childbirth (55\%); all babies were born healthy, with no complications recorded in pregnancy. 12 Women had recurrent scar ectopic (30\%). There were 2 abortion cases, 2 cases of ectopic pregnancy, and 2 cases of early miscarriage. Conclusions: Our study shows that reproductive outcomes following treatment of caesarean scar ectopic pregnancies are favourable. The risk of recurrent caesarean scar ectopic pregnancy is a concern.
\end{abstract}

\section{Keywords}

Caesarean Scar Pregnancy, Reproductive Outcomes

\section{Introduction}

Embryo implantation in a previous cesarean section scar resulting in a cesarean 
scar pregnancy (CSP) has extremely low incidence that is 1 per 3000 for the general obstetric population and 1 per 531 among those with at least 1 cesarean delivery [1]. It might be caused by the trophoblast which invades a detective and thinned out myometrium in a scar which is a remnant of previous cesarean section. Cesarean pregnancy is associated with catastrophic complications, such as uterine scar rupture and uncontrollable bleeding, which may lead to hysterectomy. So that it is important for early and accurate detection of such pregnancies. The majority of the CSPs have been diagnosed by transvaginal scan (TVS) in the early weeks of pregnancy that its criteria include 1) an empty uterine cavity, without contact with the sac, 2) a clearly visible empty cervical canal, without contact with the sac, 3) presence of the gestation sac with or without a fetal pole with or without fetal cardiac activity (depending on the gestation age) in the anterior part of the uterine isthmus, and 4) absence of or a defect in the myometrial tissue between the bladder and the sac. To reduce the risk of false diagnosis, additional information can be obtained by color flow Doppler to show distinct circular peri-trophoblastic perfusion surrounding the gestation sac or transvaginal three-dimensional (3-D) power Doppler ultrasound with combination of the multiplanar views and surface-rendered images which help to identify subtle anatomical details of a well-developed trophoblastic shell around the gestational sac. The opposite of diagnosis is the optimal management of cesarean scar pregnancy with no consensus. Generally, there are many conservative treatments that are to perform feticide prior to rupture, to remove the gestation sac and to retain patient's future fertility. Gestational age and viability, evidence of myometrial deficiency and clinical symptoms at presentation have been considered by various authors to determine the management. It is obvious that the women who have preserved their fertility may become pregnancy again while the high risk of recurrent CSP, uterine rupture and life-threatening complications still existed for those women, especially labor. In South Viet Nam, although majority of CSPs are case reports or small case series reported, the rate of conservative treatment appears to be increasing. This rate is $85.11 \%$ in applying the method of excision of trophoblastic tissues using either laparotomy, or 97.1\% when using locally and/or systemically administered methotrexate combined with surgical sac aspiration in Tu Du and Hung Vuong Hospital. Until now, we have not followed about the chances and risks of future fertility and pregnancy of cases succeeding in preservative treatment. The aim of this study was to address this issue of what happens for pregnancies of the women with whom we successfully preserved the uterus became pregnant within 2 years after termination of the scar pregnancy.

\section{Material and Methods}

This is retrospective case series of 40 patients becoming pregnancy after conservative treatment of cesarean scar pregnancy. Patients in present study were treated at Hung Vuong Hospital and Tu Du Hospital between 2015 and 2017. All electronic medical records were searched for the diagnosis of cesarean scar 
pregnancy according to code O008 of the International Classification of Diseases 10 (ICD 10). The cases are accepted into our study if there was present to diagnose CSP which based on transvaginal ultrasound using the following criteria: 1) an empty uterine cavity, without contact with the sac; 2) a clearly visible empty cervical canal, without contact with the sac; 3) presence of the gestation sac with or without a fetal pole with or without fetal cardiac activity (depending on the gestation age) in the anterior part of the uterine isthmus; 4) absence of or a defect in the myometrial tissue between the bladder and the sac.

In addition, these cases meet certain criteria as follows: 1) all detailed conservative treatment was reported and completing treatment course were confirmed; 2) having evidence that pregnancy remnants were no longer and 3) having evidence to confirm new gestation after cesarean scar pregnancy treatment.

We collected data from medical records including maternal age, gestational age at cesarean scar pregnancy diagnosis, conservative method. Delivery method, time interval between the scar pregnancy and subsequent pregnancy, and maternal and neonatal outcome were written down from both hospital medical records.

Our research was observational study. Before going research, we were accepted by Medical Committee of University of Medicine and Pharmacy at Ho Chi Minh City, Vietnam, and Tu Du Hospital. We used Stata software for describing and analyzing data.

\section{Results}

From 2015 to 2017, we searched electronic medical records with the diagnosis of cesarean scar pregnancy according to code 0008 of the International Classification of Diseases 10 (ICD 10) which treated at Hung Vương hospital and Tu Du Hospital. As of June 2019, 232 patients were successfully preserved the uterus and 44 of which became pregnancy. There were 4 cases excluded from our study because not clearly evidence of having pregnancy was given. In brief, we have intercepted and analyzed 40 cases conceive after conservative treatment of cesarean scar pregnancy.

Clinical details of 40 patients when were diagnosed cesarean scar pregnancy (CSPs) are summarized in Table 1. Average age of patient was 35 (48 \pm 4.78 years old). The gestational age at diagnosis ranged from $5+0$ to $8+0$ weeks (mean $5.8 \pm 0.9$ weeks) with an intact gestational sac. Four cases had of gestational sac containing an embryo with visible cardiac activity at $6-8$ gestational weeks and others were classified as non-viable. HCG level was so shaky with the range from 347 to $106,247 \mathrm{mIU} / \mathrm{ml}$. Twenty two of 40 (55.0\%) women received methotrexate as the primary treatment and then had surgical sac aspiration. These cases were non-viable pregnancies that were treated successfully by systemically administered MTX followed by a dilatation and curettage under ultrasound guidance. Eighteen of 40 (45.0\%) had a Foley catheter inserted and simple suction were followed. Specially, three cases of viable cesarean scar pregnancy were managed surgically without local Methotrexate. 
Table 1. Clinical details of 44 cases of cesarean scar pregnancy.

\begin{tabular}{|c|c|c|c|c|c|}
\hline \multirow{2}{*}{ Pt's file number } & \multirow{2}{*}{ Pt's age } & \multicolumn{3}{|c|}{ Pretreatment Pregnancy status } & \multirow{2}{*}{ Treatment } \\
\hline & & GA (wks) & $\mathrm{hcG}, \mathrm{mIU} / \mathrm{ml}$ & Fetus, FHR & \\
\hline TRH150 & 29 & 5 & 3158 & No & Foley + surgical sac aspiration \\
\hline NHT112 & 41 & 6.3 & 83,121 & Yes & Foley + surgical sac aspiration \\
\hline MAĐ015 & 31 & 6 & 8897 & No & MTX $^{\star}+$ surgical sac aspiration \\
\hline KIL027 & 26 & 5 & 1792 & No & Foley + surgical sac aspiration \\
\hline TRP056 & 36 & 7.1 & 59,110 & Yes & Foley + surgical sac aspiration \\
\hline NGH005 & 32 & 8.1 & 649 & No & Foley + surgical sac aspiration \\
\hline BIT020 & 43 & 6 & 3784 & No & MTX + surgical sac aspiration \\
\hline LET007 & 39 & 6 & 8609 & No & Foley + surgical sac aspiration \\
\hline NGN022 & 36 & 6 & 3758 & No & MTX + surgical sac aspiration \\
\hline OAN011 & 36 & 5 & 5478 & No & Foley + surgical sac aspiration \\
\hline CHL154 & 28 & 5 & 9925 & No & Foley + surgical sac aspiration \\
\hline HAN193 & 32 & 5 & 606 & No & Foley + surgical sac aspiration \\
\hline TRV021 & 37 & 5 & 3789 & No & MTX + surgical sac aspiration \\
\hline DUH008 & 36 & 6.1 & 8363 & No & MTX + surgical sac aspiration \\
\hline THT004 & 36 & 6 & 9687 & No & MTX + surgical sac aspiration \\
\hline CẢL016 & 32 & 6 & 36,406 & No & MTX + surgical sac aspiration \\
\hline HIH085 & 35 & 5 & 2255 & No & MTX + surgical sac aspiration \\
\hline THM046 & 42 & 6 & 29,529 & No & MTX + surgical sac aspiration \\
\hline DIT013 & 27 & 6.2 & 72,890 & No & Foley + surgical sac aspiration \\
\hline MEP017 & 40 & 5.5 & 3785 & No & Foley + surgical sac aspiration \\
\hline THN034 & 36 & 5.5 & 5038 & No & MTX + surgical sac aspiration \\
\hline PHV009 & 28 & 8 & 22,779 & No & MTX + surgical sac aspiration \\
\hline NGT003 & 41 & 5 & 527 & No & MTX + surgical sac aspiration \\
\hline MAN105 & 35 & 5 & 13,954 & No & MTX + surgical sac aspiration \\
\hline NGT006 & 33 & 5 & 5783 & No & MTX + surgical sac aspiration \\
\hline HAT038 & 34 & 5 & 656 & No & MTX + surgical sac aspiration \\
\hline NGN002 & 37 & 6.1 & 6015 & No & Foley + surgical sac aspiration \\
\hline DIN026 & 40 & 5 & 1254 & No & MTX + surgical sac aspiration \\
\hline HUN029 & 41 & 5.5 & 6028 & No & MTX + surgical sac aspiration \\
\hline THL010 & 44 & 5 & 2625 & No & MTX + surgical sac aspiration \\
\hline TRT024 & 40 & 5 & 3032 & No & Foley + surgical sac aspiration \\
\hline THN014 & 27 & 6 & 11,012 & No & Foley + surgical sac aspiration \\
\hline MIM066 & 29 & 8.3 & 106,247 & Yes & Foley + surgical sac aspiration \\
\hline HIL185 & 37 & 5 & 28,101 & No & MTX + surgical sac aspiration \\
\hline TRH057 & 33 & 6 & 25,834 & No & MTX + surgical sac aspiration \\
\hline PHH012 & 39 & 6 & 7348 & No & Foley + surgical sac aspiration \\
\hline DAT023 & 40 & 6.4 & 58,612 & No & MTX + surgical sac aspiration \\
\hline MIT082 & 39 & 8 & 14,796 & Yes & Foley + surgical sac aspiration \\
\hline NGN019 & 36 & 5 & 347 & No & MTX + surgical sac aspiration \\
\hline TRV025 & 36 & 5.5 & 29,854 & No & MTX + surgical sac aspiration \\
\hline
\end{tabular}

${ }^{*}$ MTX: Methotrexate. 
Pregnancy state of the patients after conservative treatment of cesarean scar pregnancy is shown in Table 2. The mean interval between the CSPs and subsequent pregnancy was 19.43 months (range 2.3 - 44 months) with $34 / 44$ cases being intended pregnancy and 6/44 untended pregnancies. Twenty six of 40 patients achieved normal intrauterine pregnancy, but 22 patients continued live pregnancy, 2 patients had early missed abortion and 2 decided induced abortion. Fourteen patients had ectopic pregnancy with 12 recurrent CSP.

Table 3 shows characteristics of 22 cases having alive fetus after conservative treatment of cesarean scar pregnancy. Maternal age smaller than 30 or larger than 39 years old was low while $59.09 \%$ of pts were from 30 to 39 years. Indications for elective cesarean section were prior cesarean section in 20 pts and emergency in 1 pts because of fetal distress. Of them, 1 case who uncomplicated placenta previa, was performed a cesarean delivery at $37+2$ weeks in pregnancies. Median gestational ages at delivery was $38.2 \pm 0.9$ weeks. All neonates were in good condition, with no complications which occurred during pregnancy and surgery. Especially one case was normal delivery after labor happened naturally at 37 weeks' gestation which had not uterine rupture or blood transfusion.

Besides, there were 2 cases of early missed abortion (5\%), 2 cases of ectopic pregnancy at fallopian tube (5\%) and 2 cases of induced abortion (2\%). One case of ectopic pregnancy tube was treated by methotrexate and one had laparoscopy removing pregnancy with whole tube. Two women received induced abortion due to unwanted pregnancy. CSP was excluded via ultrasound before termination.

The characteristics of women with recurrent CSP are shown in Table 4. Eleven women $(27.5 \%)$ who experienced recurrent CSP were treated successfully with reservation without any severe complications. The gestational age at diagnosis ranged from $5+0$ to $6+3$ weeks with an intact gestational sac. These women received methotrexate combined surgical sac aspiration or Foley combined aspiration. However, there was a case which was admitted urgently in serious bleeding caused by 9 weeks CSP. Open laparotomy was performed to control gestational mass and bleeding but keeping uterus with $300 \mathrm{ml}$ losing blood. Especially, one case (MIM06) had intrauterine pregnancy and recurrent CSP after treating first CSP. This woman chose induced abortion with intrauterine pregnancy.

Table 2. Pregnancy states after conservative treatment of cesarean scar pregnancy.

\begin{tabular}{cc}
\hline Characteristics & Cases $(\mathrm{n}=40)$ \\
\hline Time interval between the scar pregnancy & $19.43 \pm 15.95$ (months) \\
Subsequent pregnancy: & \\
Early missed abortion & 2.3 months; max $=44$ months $)$ \\
Ectopic pregnancy & $2(5 \%)$ \\
Recurrent CSP & $12(30 \%)$ \\
Induced abortion & $2(5 \%)$ \\
Live pregnancy & $22(55 \%)$ \\
\hline
\end{tabular}


Table 3. Outcome of pregnancy cases living after conservative treatment of cesarean scar pregnancy.

\begin{tabular}{cc}
\hline Characteristics & Cases $(\mathrm{n}=22)$ \\
\hline $\begin{array}{c}\text { Maternal age } \\
\leq 30\end{array}$ & $5(22.73 \%)$ \\
$>30-39$ & $13(59.09 \%)$ \\
$\geq 39$ & $4(18.18 \%)$ \\
Previous cesarean section & $13(59.09 \%)$ \\
2 & $9(40.91 \%)$ \\
Method of delivery & \\
Cesarean section & $21(95.45 \%)$ \\
Normal delivery & $1(4.55 \%)$ \\
Gestational age at delivery (wks) & $38.2 \pm 0.9$ \\
Placenta previa & $1(4.55 \%)$ \\
\hline
\end{tabular}

Table 4. Characteristics of 12 cases having recurrent CSPs.

\begin{tabular}{|c|c|c|c|c|c|c|c|c|}
\hline \multirow{2}{*}{ Number } & \multirow{2}{*}{ Pt's age } & \multicolumn{4}{|c|}{ Previous CSPs } & \multicolumn{3}{|c|}{ Recurrent CSPs } \\
\hline & & PARA & C/S (No.) & GA (wks.) & Treatment & Interval (mo.) & GA (wks.) & Treatment \\
\hline TRH057 & 33 & 1011 & 1 & 6 & MTX-Asp & 14 & 5 & MTX-Asp \\
\hline TRT024 & 40 & 2002 & 1 & 5 & MTX-Asp & 8 & 6 & MTX-Asp \\
\hline PHH012 & 39 & 2012 & 2 & 6 & MTX-Asp & 16 & 6 & MTX-Asp \\
\hline TUN014 & 27 & 1001 & 1 & 6 & Foley-Asp & 10 & 9 & operation \\
\hline MIM066 & 29 & 1102 & 2 & 8.3 & Foley-Asp & 10 & 5 & MTX-Asp \\
\hline MIT082 & 39 & 2002 & 2 & 8 & Foley-Asp & 33 & 6.5 & Foley-Asp \\
\hline DAT023 & 40 & 0121 & 1 & 6.4 & MTX-Asp & 23 & 6.2 & Foley-Asp \\
\hline TRV025 & 36 & 2012 & 2 & 5 & MTX-Asp & 44 & 5 & Foley-Asp \\
\hline HIL185 & 37 & 3023 & 2 & 5 & MTX-Asp & 14 & 6.4 & Foley-Asp \\
\hline NGN019 & 36 & 1001 & 1 & 5 & MTX-Asp & 35 & 6 & MTX-Asp \\
\hline THL010 & 44 & 1202 & 2 & 5 & MTX-Asp & 6 & 5 & MTX-Asp \\
\hline HUN029 & 41 & 2002 & 2 & 5 & MTX-Asp & 3 & 6 & MTX-Asp \\
\hline
\end{tabular}

MTX-Asp: Methotrexate and surgical sac aspiration; Foley-Asp: Foley and surgical sac aspiration. 


\section{Discussion}

Cesarean scar pregnancy is rare but it can cause severe maternal morbidity and mortality [2]. CSP has increased dramatically due to the increase in Cesarean section [3]. It was recently estimated that 1 in 531 women with a cesarean scar will have a CSP and that $4.2 \%$ of ectopic pregnancies are CSP [3] [4]. There are many CSP's therapies that can preserve the uterus and subsequent fertility. In our study, 40 patients had the successful conceive treatment. That success may be caused by 2 factors: 1 ) earlier detection by ultrasound to determine the localization of the CSP and 2) Foley or MTX and aspiration that was considered the first choice for management. Obviously, 40 patients were diagnosed as soon as gestational age is smaller 8 weeks so that Foley or MTX and aspiration achieved effectiveness. These patients preserving fertility and they became pregnancy whether wishing to have birth again. The important thing is still a chance of recurrence.

Until now, some case study describes pregnancy state after treatment for CSP and reported the recurrence rate of CSP in subsequent pregnancy. In J. Ben Nagi's research in 2007 [5], recurrent CSPs comprised only 5\% of cases after treatment CSP while others determined higher rate, 15.6\% [6], 11.1\% [7] and 14.3\% [8] respectively. In our present study, the recurrent rate is highest with $30 \%$ of 40 women conceiving pregnancy after treatment CSP. That recurrent rate was very high because of two reasons. Firstly, Tu Du and Hung Vuong Hospital, two largest obstetric and gynecological centers in South Viet Nam admitted more patients diagnosed with CSP. Secondly, our major method for managing CSP only resolves the gestational mass but does not repair uterine scar defect. In Qiao Wang's study, the rate of repeated CSP was higher in cases were used UAE combined with D\&C than in cases treated by laparotomy resection. That means of the five women with recurrent CSP, one was previously treated by UAE followed by laparotomy resection of CSP and repair of uterine scar defect, while the other four received combined therapy with UAE and D\&C [6]. There is, in fact, subjective in treatment selection of our cases that Foley or MTX and aspiration is the most favorable. After diagnosis was reached, the patient underwent extensive risk-benefit counseling, including a discussion of alternative therapies and a review of the risks involved. Informed consent was obtained from all patients before treatment. Individualized management strategies were adopted, depending on specific conditions of each patient. Factors, such as hemodynamic status, gestational age, serum $\beta$-hCG level, ultrasonographic findings, and patients' request, were comprehensively considered. The most common primary treatments were Foley or MTX and aspiration if gestational age smaller than 8 weeks. Second, they were followed until serum $\beta$-hCG level became negative. All of them were advised birth control for 2 years and they can get pregnancy. In short, the potential association between previous treatment and recurrent CSP could not further evaluate because our present study is observed with relatively small sample size. More prospective studies with large population are still needed.

It is important to stress that women conceiving pregnancy after treatment 
CSP are in a high risk of recurrent CSP. Obviously, 11 cases of our study followed properly the process included early sonography to confirm the intrauterine pregnancy location and reached good outcome while only one case did not do and must be controlled bleeding by emergency operation. Anyway, we lacked detecting a scar defect by trans-vaginal sonography before patient planned to have pregnancy. Seow et al. [9] reported detecting a scar defect by trans-vaginal sonography 4 years before a patient's in vitro fertilization-induced pregnancy in the scar. However, there is not enough evidence that a scar defect is cause of CSP [10]. The subsequent pregnancy after previous CSP may be chance.

Our study shows the obstetric outcomes after treatment of cesarean scar pregnancy which 22 cases gave healthy babies. Our successful pregnancy of 55\% saw the resemblance with others ranging from $60 \%$ [6] to 65\% [5]. According to our study, it seemed to be safe in women who wanted to become pregnant again, since all the successful subsequent deliveries happened in the women previously treated with medical therapy. Although we did not repair uterine scar, there were not any serious complications of having pregnancy after treating a cesarean scar pregnancy, including uterine scar rupture and internal hemorrhage, requires immediately control bleeding by hysterectomy. We decided elective cesarean section for most of cases, but one case with normal delivery did not have serious complication. This outcome was lucky. Know Min Sow [11] reported seven of the 14 patients for whom they successfully preserved the uterus became pregnant within 3 years after termination of the scar pregnancy. One patient, who became pregnant 3 months after the scar pregnancy was found, suffered uterine rupture at 38.3 weeks' gestational age. Two patients with placental accrete, and one of them who continued the existing intrauterine twin pregnancy after transvaginal sono-guided aspiration of the scar pregnancy received a cesarean hysterectomy at 32 weeks of gestation. The remaining four pregnancies were uneventful, followed by early cesarean sections at 36 weeks. Another result of Qiao Wang showed six of ten women were previously treated by UAE combined with D\&C for CSP that had successful full-term cesarean deliveries with the median blood loss during subsequent CS operation was $450 \mathrm{~mL}$ (range $300-1000 \mathrm{~mL}$ ) [6]. No placenta previa or placenta accreta was found in Qiao Wang' research. Thus, the result of subsequent pregnancies after conservative treatment of scar pregnancies are more complicated and we must pay attention to what the women decided get pregnancies again. However, we believe there are more likely to have successful pregnancy after treatment of cesarean scar pregnancy in future.

In brief, our present study is observed with relatively small sample size so that there is not enough evident to support subsequent pregnancy after previous CSP. We need more prospective studies with large population and long time for having pregnancy after conserving CSP treatment.

\section{Conclusion}

In conclusion, we reported the 2 years-follow up of reproductive outcomes after 
previous CSP treatment, with a recurrence rate of $30 \%$ and a successful pregnancy rate of 55\%. Our study provided some evidence about the safety of having pregnancy after conservative treatment of cesarean scar pregnancy by Foley or MTX and aspiration.

\section{Conflicts of Interest}

The authors declare no conflicts of interest regarding the publication of this paper.

\section{References}

[1] Ron May, M. (2011) Fertility Performance and Obstetric Outcomes among Women With Previous Cesarean Scar Pregnancy. Journal of Ultrasound in Medicine, Volume 30, 1179-1184.

https://onlinelibrary.wiley.com/doi/full/10.7863/jum.2011.30.9.1179 https://doi.org/10.7863/jum.2011.30.9.1179

[2] Chetty, J.E.M. (2009) Treating Non-Tubal Ectopic Pregnancy. Best Practice \& Research Clinical Obstetrics \& Gynaecology, 23, 529-538. https://doi.org/10.1016/j.bpobgyn.2008.12.011

[3] Ngu, V.C.S.F. (2011) Non-Tubal Ectopic Pregnancy. International Journal of Gynecology \& Obstetrics, 115, 295-297. https://doi.org/10.1016/j.ijgo.2011.07.032

[4] Fylstra, D. (2002) Ectopic Pregnancy within a Cesarean Scar: A Review. Obstetrical \& Gynecological Survey, 57, 537-543. https://doi.org/10.1097/00006254-200208000-00024

[5] Ben Nagi, J., Helmy, S., Ofili-Yebovi, D., Yazbek, J., Sawyer, E. and Jurkovic, D. (2007) Reproductive Outcomes of Women with a Previous History of Caesarean Scar Ectopic Pregnancies. Human Reproduction, 22, 2012-2015. https://doi.org/10.1093/humrep/dem078

[6] Wang, Q., Peng, H.-L., He, L. and Zhao, X. (2015) Reproductive Outcomes after Previous Cesarean Scar Pregnancy: Follow Up of 189 Women. Taiwanese Journal of Obstetrics and Gynecology, 54, 551-553. https://doi.org/10.1016/j.tjog.2015.08.006

[7] Gao, L.F., Huang, Z.W., Zhang, X., Zhou, N., Huang, X.K. and Wang, X.Y. (2016) Reproductive Outcomes Following Cesarean Scar Pregnancy-A Case Series and Review of the Literature. European Journal of Obstetrics and Gynecology and Reproductive Biology, 200, 102-107. https://doi.org/10.1016/j.ejogrb.2016.02.039

[8] Wei, L.K., Yu, L.M., Mu, R.M. and Xue, F.X. (2018) Reproductive Outcomes Following Women with Previous Cesarean Scar Pregnancy. Zhonghua Yi Xue Za Zhi, 98, 2194-2197.

[9] Seow, K.-M., Hwang, J.-L. and Tsai, Y.-L. (2001) Ultrasound Diagnosis of a Pregnancy in a Cesarean Section Scar. Ultrasound in Obstetrics \& Gynecology, 18, 547-551. https://doi.org/10.1046/j.0960-7692.2001.00569.x

[10] Seow, K.-M., Hwang, J.-L., Tsai, Y.-L., Huang, L.-W., Lin, Y.-H. and Hsieh, B.-C. (2009) Subsequent Pregnancy Outcome after Conservative Treatment of a Previous Cesarean Scar Pregnancy. Acta Obstetricia et Gynecologica Scandinavia, 83, 1167-1172.

[11] Jurkovic, D., Hillaby, K., Woelfer, B., Lawrence, A., Salim, R. and Elson, C.J. (2003) First-Trimester Diagnosis, and Management of Pregnancies Implanted into the Lower Uterine Segment Cesarean Section Scar. Ultrasound in Obstetrics \& Gynecology, 21, 220-227. https://doi.org/10.1002/uog.56 